\title{
All-trans retinoic acid promotes macrophage phagocytosis and decreases inflammation via inhibiting CD14/TLR4 in acute lung injury
}

\author{
SHUANGXUE LI ${ }^{1}$, YUANSHENG LEI ${ }^{2}$, JIEYUN LEI ${ }^{3}$ and HUI LI ${ }^{4}$ \\ ${ }^{1}$ Department of Respiratory and Critical Care Medicine, Shanxi Provincial People's Hospital, Taiyuan, Shanxi 030012; \\ ${ }^{2}$ Department of Neurology, The Second Hospital of Shanxi Medical University, Taiyuan, Shanxi 030001; \\ ${ }^{3}$ Department of Cardiology, Taiyuan Central Hospital, Taiyuan, Shanxi 030009; ${ }^{4}$ Department of Gynecology, \\ Shanxi Provincial People's Hospital, Taiyuan, Shanxi 030012, P.R. China
}

Received September 18, 2020; Accepted February 18, 2021

DOI: $10.3892 / \mathrm{mmr} .2021 .12508$

\begin{abstract}
Acute lung injury (ALI) is a common clinical emergency and all-trans retinoic acid (ATRA) can alleviate organ injury. Therefore, the present study investigated the role of ATRA in ALI. Lipopolysaccharide (LPS)-induced ALI rats were treated with ATRA and the arterial partial pressure of oxygen $\left(\mathrm{PaO}_{2}\right)$, lung wet/dry weight (W/D) ratio and protein content in the bronchial alveolar lavage fluid (BALF) were measured to evaluate the effect of ATRA on ALI rats. Alveolar macrophages were isolated from the BALF. The phagocytic function of macrophages was detected using the chicken erythrocyte phagocytosis method and flow cytometry. The viability of macrophages was measured using a Cell Counting Kit- 8 assay, and apoptosis was analyzed using a TUNEL assay and flow cytometry. The expression levels of Toll-like receptor 4 (TLR4) and cluster of differentiation (CD)14 on the macrophage membrane were detected by immunofluorescence staining. The protein levels of TLR4, CD14, phosphorylated (p)-65, p65, p-ІкB $\alpha$ and IкB $\alpha$ were analyzed using western blotting. The concentrations of IL-6, IL- $1 \beta$ and macrophage inflammatory protein- 2 in the plasma of rats were detected by ELISA. Macrophages were treated with IAXO-102 (TLR4 inhibitor) to verify the involvement of CD14/TLR4 in the effect of ATRA on ALI. ATRA provided protection against LPS-induced ALI, as evidenced by the increased $\mathrm{PaO}_{2}$ and reduced lung W/D ratio and protein content in the BALF. ATRA enhanced macrophage phagocytosis and viability and reduced apoptosis and inflammation
\end{abstract}

Correspondence to: Professor Hui Li, Department of Gynecology, Shanxi Provincial People's Hospital, 29 Shuangta Temple Street, Taiyuan, Shanxi 030012, P.R. China

E-mail: lihui05250@163.com

Key words: acute lung injury, all-trans retinoic acid, cluster of differentiation 14, Toll-like receptor 4, macrophage, phagocytosis, inflammation in ALI rats. Mechanically, ATRA inhibited CD14 and TLR4 expression and NF- $\mathrm{KB}$ pathway activation. ATRA enhanced macrophage phagocytosis and reduced inflammation by inhibiting the CD14/TLR4-NF- $\kappa$ B pathway in LPS-induced ALI. In summary, ATRA inactivated the NF- $\kappa B$ pathway by inhibiting the expression of CD14/TLR4 receptor in the alveolar macrophages of rats, thus enhancing the phagocytic function of macrophages in ALI rats, improving the activity of macrophages, inhibiting apoptosis, reducing the levels of inflammatory factors, and consequently playing a protective role in ALI model rats. This study may offer novel insights for the clinical management of ALI.

\section{Introduction}

Acute lung injury (ALI), a severe heterogeneous clinical syndrome of lung injury, is mainly responsible for most acute respiratory symptoms (1). Pathologically, it is often characterized by diffuse alveolar injury and regional alveolar hypoxia $(1,2)$. Acute inflammation is closely implicated in the pathogenesis of ALI (3). The diagnosis of ALI is usually based on clinical and imaging criteria with great diagnostic uncertainty (1). Currently, standard therapies for ALI, such as prone position and neuromuscular blockade, are mainly focused on lung protection, but with limited efficacy $(4,5)$. Therefore, novel diagnostic markers and effective treatments for ALI at the molecular level are required to be explored from a pathophysiological perspective.

Vitamin A and its metabolites such as retinoic acid (RA) and all-trans RA (ATRA) serve a vital role in maintaining the balance of immune responses and regulating developmental abnormalities, such as respiratory system defects and lung agenesis $(6,7)$. As an endocrine hormone, ATRA can trigger genomic effects (8). Compelling evidence reveals that ATRA can effectively alleviate organ injury, including kidney and liver injury $(9,10)$. In addition, ATRA can relieve chronic inflammation-induced emphysema characterized by alveolar wall destruction (11). However, the effect of ATRA on ALI remains to be elucidated.

Alveolar macrophages, a type of tissue-resident macrophage, can regulate pathogen-induced immune responses and 
contribute to maintaining the homeostasis of the lung (12). Alveolar macrophages possess powerful phagocytosis that can keep the airway free of bacteria and foreign particles, thereby protecting against lung injury (13). In addition, alveolar macrophages are intimately associated with lung inflammation (14). Mechanically, the role of macrophages is mediated by a series of receptors on their surface, of which toll-like receptor 4 (TLR4) and cluster of differentiation (CD) 14 are the two most important (15).

Therefore, it is reasonable to hypothesize that ATRA serves a vital role in lipopolysaccharide (LPS)-induced ALI with the involvement of CD14 and TLR4 in macrophages. Consequently, the present study sought to identify the role of ATRA in ALI and to explore the related regulatory mechanism, which may provide novel insights into the therapies for ALI.

\section{Materials and methods}

Ethics statement. All animal procedures were performed in accordance with the guidelines of the Animal Ethics Committee of Shanxi Provincial People's Hospital and ethics approval was received (approval no. 2019-56). Significant efforts were made to minimize animal numbers and their suffering.

Bioinformatics analysis. Signaling pathway enrichment analysis was performed using the Kyoto Encyclopedia of Genes and Genomes (KEGG) database (https://www.kegg.jp).

Establishment of the ALI rat model and rat grouping. A total of 30 adult male Sprague-Dawley (SD) rats (8-10 weeks; 220-270 g) were provided by Shanghai SLAC Laboratory Animal Co., Ltd. [SYXK (Shanghai) 2018-0038]. Rats were placed in a clean environment $\left(20^{\circ} \mathrm{C} ; 50-60 \%\right.$ humidity) and raised under a 12-h light/dark cycle. All animals were euthanized by an intraperitoneal injection of pentobarbital sodium ( $\geq 100 \mathrm{mg} / \mathrm{kg}$ ).

SD rats were randomly allocated to the ALI, ATRA and control groups, with 10 rats in each group. LPS solution (10 mg/ml; Sigma-Aldrich; Merck KGaA) was prepared with normal saline. Rats in the ALI and ATRA groups were injected with $5 \mathrm{mg} / \mathrm{kg}$ LPS solution via the caudal vein. In addition, 5 days before LPS injection, rats in the ALI group were administered olive oil $(0.5 \mathrm{ml} / \mathrm{kg} / \mathrm{time})$ by gavage once a day and then intraperitoneally injected with $1 \mathrm{ml} / \mathrm{kg}$ olive oil after LPS injection. Meanwhile, 5 days before LPS injection, rats in the ATRA group were administered olive oil containing $30 \mathrm{mg} / \mathrm{kg}$ ATRA $(0.5 \mathrm{ml} / \mathrm{kg} /$ time $)$ by gavage once a day and then intraperitoneally injected with $1 \mathrm{ml} / \mathrm{kg}$ olive oil containing $5 \mathrm{mg} / \mathrm{kg}$ ATRA. Rats in the control group were administered equal amount of normal saline for 7 days. On day 7,24 h after an intraperitoneal injection of ATRA, rats in each group were euthanized and sampled (16).

Detection of arterial partial pressure of oxygen $\left(\mathrm{PaO}_{2}\right)$. The rats were anesthetized by intraperitoneal injection of $50 \mathrm{mg} / \mathrm{kg}$ pentobarbital sodium (17). Then, $2 \mathrm{ml}$ blood was collected from the external carotid artery using a blood gas needle. Blood gas was analyzed using an ABL 700 Radiometer (Radiometer Trading) blood gas analyzer.
Detection of lung wet/dry weight (W/D) ratio. After the rats were euthanized, the lung tissues were removed, and water and blood were removed from the surface of the lung tissues. The lung tissues were weighed using an electronic balance (wet weight), wrapped in tin foil paper, dried in an oven at $70^{\circ} \mathrm{C}$ for $72 \mathrm{~h}$ and weighed (dry weight). The W/D ratio of the lung tissues was calculated.

Detection of the protein content in the bronchial alveolar lavage fluid $(B A L F)$. After the rats were euthanized, the lung tissues of rats in each group were removed and subjected to lavage three times repeatedly with phosphate-buffered saline at $4^{\circ} \mathrm{C}$ and $5 \mathrm{ml}$ each time with a total perfusion amount of $15 \mathrm{ml} /$ rat and then BALF was harvested. Following centrifugation $\left(4^{\circ} \mathrm{C} ; 2,500 \mathrm{x} \mathrm{g} ; 10 \mathrm{~min}\right)$, the supernatant was collected for the determination of the total protein level using the bicinchoninic acid (BCA) kit (Thermo Fisher Scientific, Inc.) (18).

Hematoxylin and eosin $(H \& E)$ staining. The right lungs of rats were immersed in formalin at $25^{\circ} \mathrm{C}$ for $24 \mathrm{~h}$, embedded in paraffin and sliced into $4-\mu \mathrm{m}$-thick sections. The sections were then dewaxed in xylene at $25^{\circ} \mathrm{C}$ and rehydrated with gradient ethanol. The sections were subsequently stained with $100 \mu 1$ hematoxylin solution (cat. no. PT001; Shanghai Bogoo Biological Technology Co., Ltd.) for $10 \mathrm{~min}$ and eosin solution (cat. no. G1424; Beijing Solarbio Science \& Technology Co., Ltd.) for $3 \mathrm{~min}$, both at $25^{\circ} \mathrm{C}$. The sections were observed and photographed under a light microscope (magnification, x200). The lung injury score was assessed according to the American Thoracic Society ALI pathological score system (19). Lung injury included the following categories: Alveolar congestion, bleeding, infiltration or aggregation of interstitial neutrophils or neutrophils in the vessel wall, alveolar septum thickening, or hyaline membrane formation. It was classified into four grades: 0 , no or very slight injury; 1 , mild injury; 2 , moderate injury; 3 , severe injury; and 4 , very severe injury. The total score of cumulative lesions was used as the pathological score for ALI. A high score indicated serious injury (19).

Preparation and grouping of the macrophages. A total of three rats were randomly selected from each group. The BALF was collected and centrifuged $\left(20^{\circ} \mathrm{C} ; 2,000 \mathrm{x} \mathrm{g}\right.$; $30 \mathrm{~min}$ ) and the precipitate harvested. The cells were resuspended in RMPI-1640 medium (Thermo Fisher Scientific, Inc.) containing $10 \%$ fetal bovine serum (Sangon Biotech Co., Ltd.). The cell suspension was placed in a glass culture dish and incubated for $2 \mathrm{~h}\left(37^{\circ} \mathrm{C} ; 5 \% \mathrm{CO}_{2} ; 95 \%\right.$ humidity). The cell suspension was removed and the culture dish was repeatedly washed with normal saline. The washing solution containing adherent cells was collected and centrifuged at $4^{\circ} \mathrm{C}$ and $250 \mathrm{x}$ g for $10 \mathrm{~min}$ to obtain alveolar macrophages. The cell concentration was adjusted to $1 \times 10^{6}$ cells/ml using PBS for subsequent experiments (20).

The macrophages extracted from normal, ALI and ATRA-treated ALI rats were grouped into control, ALI and ATRA groups, respectively. The TLR4 inhibitor IAXO-102 $(10 \mu \mathrm{M})$ (MedChemExpress) was added to the ALI and ATRA groups for interference, with the addition of PBS as control, which were recorded as ALI + PBS, ALI + IAXO-102, ATRA + PBS and ATRA + IAXO-102 groups, respectively. 
Detection of myeloperoxidase (MPO) activity. Macrophages were re-suspended in a 4-fold volume of MPO buffer and then centrifuged $\left(4^{\circ} \mathrm{C} ; 13,000 \mathrm{~g} ; 10 \mathrm{~min}\right)$. The supernatant was collected, transferred to a clean tube and placed on ice. MPO activity was determined using the MPO activity assay kit (cat. no. ab105136, Abcam). The optical density (OD) at $460 \mathrm{~nm}$ was determined using a microplate reader (Bio-Rad Laboratories, Inc.).

Detection of the phagocytic function of the macrophages. The chicken erythrocyte phagocytosis method was used. Briefly, the prepared macrophages were suspended in culture medium and mixed well. Following the addition of Hanks solution, the macrophages were evenly mixed with an appropriate amount of chicken erythrocytes (Thermo Fisher Scientific, Inc.). The ratio of macrophages to chicken erythrocytes was adjusted to approximately $1: 600$. The mixture was incubated at $37^{\circ} \mathrm{C}$ for $0.5 \mathrm{~h}$ after being agitated and mixed every $10 \mathrm{~min}$ and then centrifuged $\left(25^{\circ} \mathrm{C} ; 1,000 \mathrm{x} \mathrm{g} ; 10 \mathrm{~min}\right)$. Afterwards, the cell suspension was dropped onto a slide for a cell suspension smear preparation. The slides were fixed using acetone and methanol (1:1) at room temperature for $10 \mathrm{~min}$ and then Giemsa staining solution was added for an 8-min incubation at room temperature. The number of macrophages phagocytizing chicken erythrocytes in 100 macrophages was observed under a light microscope (magnification, x400). The phagocytic rate $=$ the number of macrophages phagocytizing chicken erythrocytes in 100 macrophages/100 and the phagocytic index $=$ the total number of chicken erythrocytes phagocytized by 100 macrophages/100.

Flow cytometry was performed. Macrophages in each group were seeded onto 12 -well plates $\left(5 \times 10^{4}\right.$ cells/well) and $1 \mu \mathrm{l}$ fluorescein isothiocyanate (FITC)-labeled fluorescent microspheres $\left(4.55 \times 10^{7}\right.$ microspheres with a diameter of $\left.1 \mu \mathrm{m}\right)$ was added to each well for $2-\mathrm{h}$ at $37^{\circ} \mathrm{C}$. Following removal of the culture supernatant, macrophages were detached with $0.25 \%$ trypsin, washed three times with PBS and fixed with $4 \%$ paraformaldehyde at room temperature for $20 \mathrm{~min}$. The mean fluorescence intensity (MFI) of the macrophages and the percentage of phagocytizing fluorescent microspheres (phagocytic fluorescent microspheres) were measured using a flow cytometer (MoFlo Astrios EQ; Beckman Coulter, Inc.). Higher MFI values and percentages of phagocytic positive cells indicated a stronger phagocytic ability.

Reverse transcription quantitative $(R T-q) P C R$. Total RNA was extracted using TRIzol ${ }^{\circledR}$ reagent (Invitrogen; Thermo Fisher Scientific, Inc.) and reverse transcribed into cDNA using the PrimeScript RT kit (cat. no. RR037A, Takara Bio, Inc.). The reaction volume was $10 \mu \mathrm{l}$. The reaction solution was then subjected to fluorescence qPCR using the SYBR ${ }^{\circledR}$ Premix ExTaq II kit (cat. no. RR820A, Takara Bio, Inc.) on a fluorescent qPCR instrument (ABI 7500; Applied Biosystems; Thermo Fisher Scientific, Inc.). The following thermocycling conditions were used for the qPCR: Initial denaturation for $1 \mathrm{~min}$ at $95^{\circ} \mathrm{C}$; followed by 40 cycles of $15 \mathrm{sec}$ at $95^{\circ} \mathrm{C}, 30 \mathrm{sec}$ at $55^{\circ} \mathrm{C}$, and $35 \mathrm{sec}$ at $72^{\circ} \mathrm{C}$; followed by a final extension for $30 \mathrm{sec}$ at $95^{\circ} \mathrm{C}$ and $35 \mathrm{sec}$ at $55^{\circ} \mathrm{C}(21)$. Relative expression of genes was examined using the $2^{-\Delta \Delta \mathrm{Cq}}$ method (22), with GAPDH as the internal reference. The experiments were repeated three times. The experiment was repeated three times. The primers (Table I) were synthesized by Sangon Biotech Co., Ltd.

Cell Counting Kit-8 (CCK-8) assay. Macrophage cells $(5,000$ cells/well) in each group were seeded onto 96 -well plates. After $48 \mathrm{~h}$ of culture, cell viability was measured according to the manufacturer's instructions of the CCK-8 detection kit (R\&D Systems, Inc.). The OD value at $450 \mathrm{~nm}$ of each well was detected.

TUNEL staining. The macrophages were rinsed with PBS for $5 \mathrm{~min}$, fixed in $4 \%$ paraformaldehyde at room temperature for $30 \mathrm{~min}$ and washed with PBS three times ( $5 \mathrm{~min} / \mathrm{time})$. Next, the macrophages were permeated with $0.1 \%$ Triton $\mathrm{X}-100$ for $10 \mathrm{~min}$, rinsed twice with PBS (5 min/time) and then added to the TUNEL detection solution (Sigma-Aldrich; Merck KGaA) for incubation $\left(37^{\circ} \mathrm{C} ; 60 \mathrm{~min}\right)$ in the dark. After being washed with PBS for $5 \mathrm{~min}$, the macrophages were stained with $5 \mathrm{mg} / \mathrm{l}$ 4',6-diamidino-2-phenylindole (DAPI) for $10 \mathrm{~min}$ at room temperature and washed with PBS three times (5 min/time). An anti-fade mounting medium was used for sealing. The images were collected under a fluorescence inverted microscope (Olympus Corporation). A total of five visual fields (magnification, x200) were randomly selected. The images were analyzed using Image-Pro Plus 6.0 (Media Cybernetics, Inc.) and the apoptosis rate was calculated.

Detection of apoptosis using flow cytometry. Cell apoptosis was analyzed using an Annexin-V-FITC cell apoptosis detection kit (BioVision Inc.), according to the manufacturer's protocol. Annexin-V-FITC, propidium iodide (PI) and HEPES buffer solution were prepared as the Annexin-V-FITC/PI staining solution at the ratio of 1:2:50. Macrophages were detached using trypsin, washed with PBS and stained with Annexin-V-FITC/PI staining solution at room temperature for $15 \mathrm{~min}$ in the dark. The apoptosis rate was detected using a flow cytometer (MoFlo Astrios EQ, Beckman Coulter, Inc.). The lower left quadrant (Q4) on the scatter plot showed healthy living cells; the upper left quadrant (Q1) showed mechanically damaged cells; the lower right quadrant (Q3) showed early apoptotic cells and the upper right quadrant (Q2) showed necrotic and late apoptotic cells. The apoptotic rate=early apoptosis percentage $(\mathrm{Q} 3)+$ late apoptosis percentage $(\mathrm{Q} 2)$.

Enzyme-linked immunosorbent assay (ELISA). Carotid artery blood was collected for the assessment of IL-6 (cat. no. DY406), IL-1 $\beta$ (cat. no. MLB00C) and macrophage inflammatory protein-2 (MIP-2; cat. no. 1084-M2) in plasma using ELISA kits (R\&D Systems, Inc.). The OD value at $450 \mathrm{~nm}$ was determined.

Immunofluorescence. The frozen tissues $\left(-15^{\circ} \mathrm{C}\right)$ were sectioned $(10-\mu \mathrm{m}$ thick) using a freezing microtome. The frozen sections were washed with PBS and the membranes were permeated with $0.3 \%$ Triton $\mathrm{X}-100(50 \mu \mathrm{l})$ by gavage at room temperature for $15 \mathrm{~min}$. The antigen was extracted using sodium citrate solution and the sections were incubated with normal goat serum (Sangon Biotech Co., Ltd.). The sections were cultured with the antibodies against TLR4 (cat. no. ab13556, 1:500, Abcam) and CD14 (cat. no. ab183322, 1:100, Abcam) at $4^{\circ} \mathrm{C}$ overnight. 
Table I. Primer sequences used for reverse transcriptionquantitative PCR.

\begin{tabular}{ll}
\hline Gene & \multicolumn{1}{c}{ Primer sequence $\left(5^{\prime} \rightarrow 3^{\prime}\right)$} \\
\hline Toll-like & F: ACAAGGCATGGCATGGCTTACAC \\
receptor 4 & R: TGTCTCCACAGCCACCAGATTCTC \\
CD14 & F: ACTTCTCAGATCCGAAGCCAG \\
& R: CCGCCGTACAATTCCACAT \\
IкB & F: GCTGAAGAAGGAGCGGCTACT \\
& R: TCGTACTCCTCGTCTTTCATGGA \\
P65 & F: ACAACCCCTTCCAAGTTCCT \\
& R: ATCTTGAGCTCGGCAGTGTT \\
GAPDH & F: GGGTGATGCTGGTGCTGAGTATGT \\
& R: AAGAATGGGAGTTGCTGTTGAAGT
\end{tabular}

F, forward; R, reverse.

After $1 \mathrm{~h}$ of incubation at room temperature, the sections were cultured with goat anti-rabbit antibody immunoglobulin $\mathrm{G}$ (IgG) (cat. no. ab205718, 1:2,000, Abcam) containing FITC for $1 \mathrm{~h}$ at room temperature. The nuclei were stained with DAPI at room temperature for $5 \mathrm{~min}$ and the cells were observed under a Biorevo BZ9000 fluorescence microscope (Keyence Corporation; magnification, $\mathrm{x} 200$ ) in five randomly selected fields of view.

Western blot (WB analysis). Total protein was extracted using RIPA lysis buffer containing phenylmethanesulfonyl fluoride (Beyotime Institute of Biotechnology). Protein concentration was determined using the BCA protein quantitative kit (Wuhan Boster Biological Technology, Ltd.) and $50 \mu \mathrm{g}$ protein/lane was separated by $10 \%$ SDS-PAGE, transferred to polyvinylidene fluoride membranes and blocked with $5 \%$ bovine serum albumin (Sangon Biotech Co., Ltd.) at room temperature for $2 \mathrm{~h}$ to block nonspecific binding. The diluted primary antibodies rabbit anti-mouse TLR4 (cat. no. ab217274, 1:300, Abcam), CD14 (cat. no. ab221678, 1:1,000, Abcam), P65 (cat. no. ab16502, 1:1,000, Abcam), p-P65 (cat. no. ab194726,

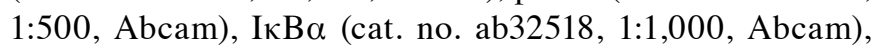
p-IкB $\alpha$ (cat. no. ab133462, 1:1,000, Abcam) and $\beta$-actin (cat. no. ab8227, 1:2,000, Abcam) were added for overnight incubation at $4^{\circ} \mathrm{C}$. The membranes were washed and then cultured with horseradish peroxidase-labeled goat anti-rabbit IgG (cat. no. ab205718, 1:2,000, Abcam) for $1 \mathrm{~h}$ at room temperature. An enhanced chemiluminescence working solution (EMD Millipore) was used for membrane development. Image-Pro Plus 6.0 (Media Cybernetics, Inc.) was used to quantify the gray value of bands in $\mathrm{WB}$ images. $\beta$-actin served as the internal control.

Statistical analysis. All data were analyzed using SPSS v21.0 (IBM Corp.). First, the normality and homogeneity of variance tests verified that the data were normally distributed and showed homogeneity of variance. The measurement data were represented as mean \pm standard deviation. Comparison between two groups was performed using an independent sample t-test. Comparisons among groups were analyzed using one-way or two-way analysis of variance followed by Tukey's multiple comparisons test. $\mathrm{P}<0.05$ was considered to indicate a statistically significant difference.

\section{Results}

ATRA exhibits protective effects on LPS-induced ALI rats. The rat model of ALI was induced by LPS injection. Compared with the ALI + saline group, there were no significant differences in $\mathrm{PaO}_{2}, \mathrm{BALF}$ protein content and W/D ratio in the ALI + olive group (Supplementary materials and methods; Supplementary results; Fig. S1). In addition, $5 \mathrm{mg} / \mathrm{kg}$ ATRA demonstrated an improved protective effect on ALI rats following LPS injection (Supplementary materials and methods; Fig. S2). Therefore, $5 \mathrm{mg} / \mathrm{kg}$ ATRA was used in the following experiment. The arterial blood and BALF of ALI rats and control rats at $24 \mathrm{~h}$ after modeling were collected to determine $\mathrm{PaO}_{2}$ concentration and protein content. ALI rats showed significantly increased protein content in BALF and decreased $\mathrm{PaO}_{2}$ concentration (both $\mathrm{P}<0.01$; Fig. $1 \mathrm{~A}$ and $\mathrm{B}$ ). The lung W/D ratio was measured, and it was observed that the lung W/D ratio was significantly increased in ALI rats $(\mathrm{P}<0.01$; Fig. 1C). The above results indicated that the ALI rat model was successfully induced.

ATRA has a variety of biological functions, including affecting the cell proliferation cycle, immune cell functional molecule expression and various cytokine secretions (23). However, the effect and mechanism of ATRA in LPS-induced ALI rats require further study. Therefore, LPS-induced ALI rats were treated with ATRA. ATRA treatment markedly increased $\mathrm{PaO}_{2}$ concentration and reduced protein content and lung W/D ratio (all $\mathrm{P}<0.05$; Fig. $1 \mathrm{~A}-\mathrm{C}$ ). Additionally, the pathological changes in lung tissues were measured using $\mathrm{H} \& \mathrm{E}$ staining to assess the tissue injury. The results revealed that ALI rats exhibited abundant inflammatory cells in the alveolar cavity of lung tissues, massive exudate and clearly dilated and congested pulmonary interstitial capillaries $(\mathrm{P}<0.01)$, while ATRA treatment effectively reversed all these trends $(\mathrm{P}<0.01$; Fig. 1D). The lung injury score of rats in the ATRA group was significantly reduced (Fig. 1E). Thus, ATRA possessed protective effects in LPS-induced ALI rats.

ATRA enhances macrophage phagocytosis in ALI rats. A previous study hypothesized that the phagocytic function of macrophages is crucial for maintaining tissue homeostasis and is closely associated with inflammation and immune response (24). Therefore, it was important to explore the function of alveolar macrophages in ALI. BALF was collected and alveolar macrophages were isolated and purified. MPO activity of alveolar macrophages was measured using an MPO kit. As demonstrated by the results, macrophages in the ALI group showed noticeably increased MPO activity compared with the control cells, which then decreased after ATRA treatment (both $\mathrm{P}<0.01$; Fig. $2 \mathrm{~A}$ ). Next, the phagocytic rate and phagocytic index of macrophages were detected using the chicken erythrocyte phagocytosis method. It was observed that the phagocytic rate and phagocytic index were notably decreased in ALI-induced macrophages and ATRA treatment effectively enhanced the phagocytic rate and index of alveolar macrophages (all $\mathrm{P}<0.01$; Fig. $2 \mathrm{~B}$ and $\mathrm{C}$ ). Flow cytometry 
A

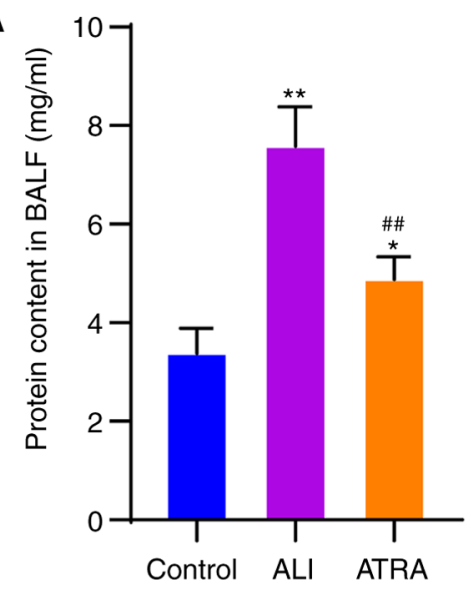

B

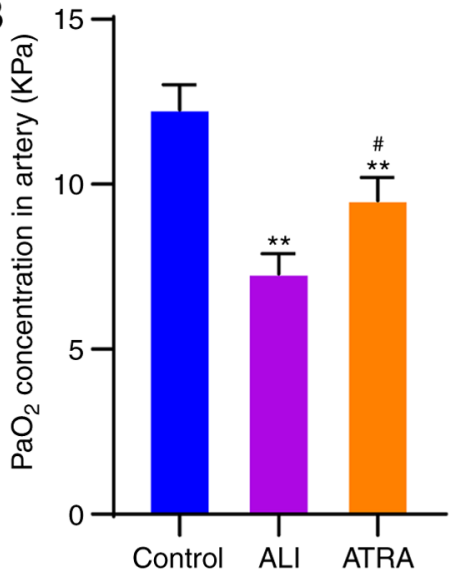

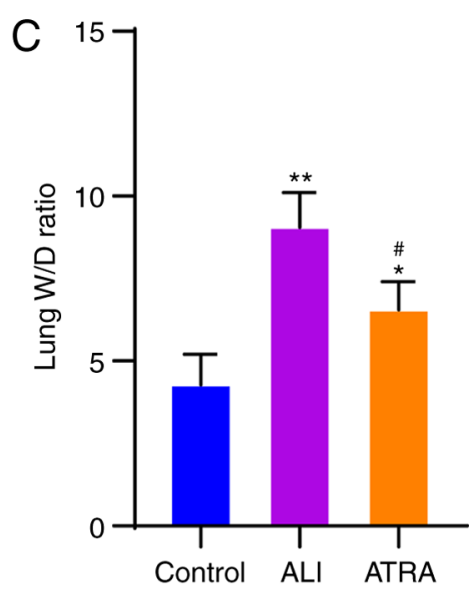

E

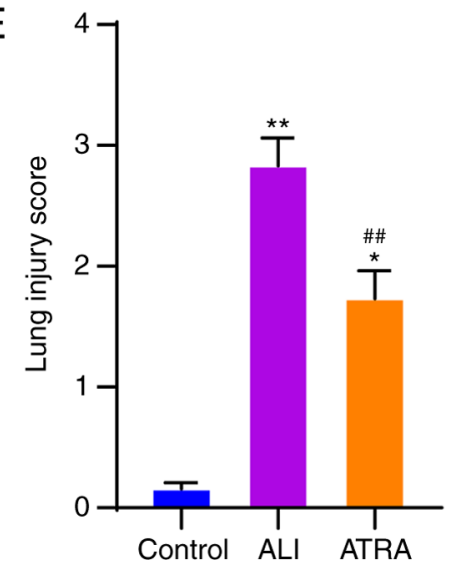

Figure 1. ATRA possesses protective effects on LPS-induced ALI rats. (A) Protein content in BALF detected using a kit. (B) $\mathrm{PaO}_{2}$ in arterial blood detected using a blood gas analyzer. (C) Lung W/D ratio. (D) Lung tissue injury observed with hematoxylin and eosin staining. Magnification, x200. (E) Lung injury score assessed with high score indicative of severe injury. $\mathrm{N}=10$ in each group. A total of three independent experiments were repeatedly conducted and the data are expressed as mean \pm standard deviation. Data analyzed using one-way analysis of variance, followed by Tukey's multiple comparisons test. " $\mathrm{P}<0.05$, ${ }^{* *} \mathrm{P}<0.01$ vs. control group; ${ }^{\mathrm{P}} \mathrm{P}<0.05,{ }^{\# \#} \mathrm{P}<0.01$ vs. ALI group. ATRA, all-trans retinoic acid; LPS, lipopolysaccharide; ALI, acute lung injury; BALF, bronchial alveolar lavage fluid; $\mathrm{PaO}_{2}$, arterial partial pressure of oxygen; W/D, wet/dry weight.

was used to detect phagocytosis of fluorescent microspheres by macrophages. It was found that macrophages in the ALI group exhibited clearly reduced MFI values and percentage of phagocytic positive cells and ATRA treatment partially reversed these trends (all $\mathrm{P}<0.01$; Fig. $2 \mathrm{D}$ and $\mathrm{E}$ ). Briefly, it was demonstrated that ATRA enhanced the phagocytic function of macrophages in ALI rats.

ATRA increases viability and inhibits the apoptosis of macrophages and inflammation in ALI rats. The effects of ATRA on the viability and apoptosis of macrophages in ALI rats were assessed. CCK-8 assay revealed that ATRA treatment markedly increased the viability of macrophages (all $\mathrm{P}<0.01$; Fig. 3A). According to TUNEL and flow cytometry, macrophages in the ALI group showed markedly elevated apoptosis, which was then notably reduced after ATRA treatment (all $\mathrm{P}<0.01$; Fig. 3B and $\mathrm{C}$ ).

Macrophage activation is closely associated with inflammatory responses (25). Hence, levels of inflammatory cytokines (IL-6, IL-1 $\beta$ and MIP-2) in the plasma of rats in each group were detected using ELISA kits. ATRA-treated ALI rats exhibited noticeably reduced levels of inflammatory cytokines (all $\mathrm{P}<0.01$; Fig. 3D-F). These results revealed that ATRA increased the viability and inhibited apoptosis of macrophages and decreased inflammatory cytokine levels in ALI rats.

\section{ATRA inhibits CD14 and TLR4 upregulation and downstream} $N F-\kappa B$ pathway activation in LPS-induced ALI rat macrophages. CD14 and TLR4 are important receptors in the LPS signal transduction pathway (26). Therefore, CD14 and TLR4 expression levels on the macrophage membrane were detected using immunofluorescence and CD14 and TLR4 protein levels were detected using WB. CD14 and TLR4 expression levels were clearly upregulated in macrophages in the ALI group and notably decreased following ATRA treatment (Fig. 4A). RT-qPCR and WB results further confirmed the above results (all $\mathrm{P}<0.01$; Fig. $4 \mathrm{C}$ and $\mathrm{D}$ ). Briefly, it was demonstrated that ATRA treatment inhibited CD14 and TLR4 expressions in macrophages. Furthermore, according to the KEGG database, there are many pathways downstream of CD14/TLR4 receptors (Fig. 4B), among which the NF- $\kappa \mathrm{B}$ pathway serves a key role in ALI $(27,28)$. Therefore, the mRNA and proteins of the NF- $\kappa$ B pathway were detected using RT-qPCR and WB. The mRNA expression of P65 and $\mathrm{I} \kappa \mathrm{B} \alpha$ was increased in macrophages in the ALI group and the protein ratios of $\mathrm{p}-\mathrm{P} 65 / \mathrm{P} 65$ and $\mathrm{p}-\mathrm{I} \kappa \mathrm{B} \alpha / \mathrm{I} \kappa \mathrm{B} \alpha$ were also clearly 

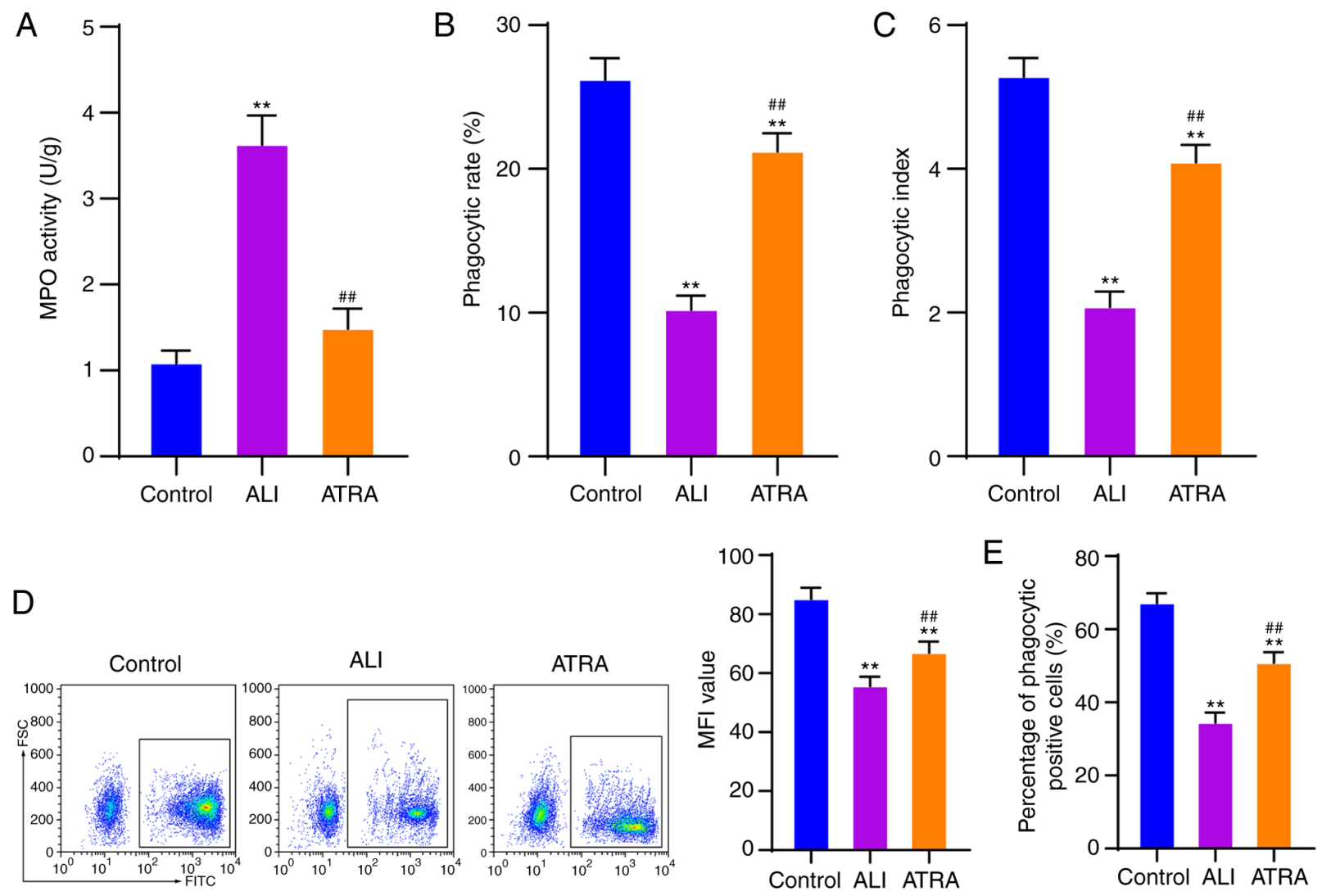

Figure 2. ATRA enhances macrophage phagocytosis in ALI rats. (A) MPO activity of alveolar macrophages was measured. (B) Phagocytic rate and (C) phagocytic index of macrophages were detected using the chicken erythrocyte phagocytosis method. (D) MFI value of macrophages and (E) the percentage of phagocytic positive cells were detected using flow cytometry. The cell experiments were repeated three times independently. Data are expressed as mean \pm standard deviation and analyzed using one-way analysis of variance, followed by Tukey's multiple comparisons test. ${ }^{* *} \mathrm{P}<0.01 \mathrm{vs}$. control group; ${ }^{\# \#} \mathrm{P}<0.01$ vs. ALI group. ATRA, all-trans retinoic acid; ALI, acute lung injury; MPO, myeloperoxidase; MFI, mean fluorescence intensity.

elevated, while ATRA treatment effectively reduced these mRNA expressions and protein ratios (all $\mathrm{P}<0.01$; Fig. 4C). Taken together, ATRA inhibited CD14/TLR4 expression and $\mathrm{NF}-\kappa \mathrm{B}$ pathway activation in macrophages in LPS-induced ALI rats.

ATRA enhances macrophage phagocytosis and reduces inflammation via inhibiting CD14/TLR4 in ALI rats. To clarify the specific regulatory mechanism of ATRA in macrophage phagocytosis in ALI rats, the ALI group (ALI + IAXO-102) and ATRA group (ATRA + IAXO-102) were treated with the TLR4 inhibitor IAXO-102, with the addition of PBS as the control. TLR4 expression, and mRNA expression and protein levels of the NF- $\mathrm{kB}$ pathway were detected using RT-qPCR and WB. The results revealed that TLR4 expression, mRNA expression of $\mathrm{P} 65$ and $\mathrm{I} \kappa \mathrm{B} \alpha$, and protein levels of p-P65/P65 and $\mathrm{p}-\mathrm{I} \kappa \mathrm{B} \alpha / \mathrm{I} \kappa \mathrm{B} \alpha$ were notably decreased after the addition of TLR4 inhibitor IAXO-102 (all P<0.01; Fig. 5A and B). Compared with those in the ALI + PBS group, macrophages in the ALI + IAXO-102 group and the ATRA + PBS group showed clearly decreased MPO activity, and macrophages in the ATRA + IAXO-102 group exhibited the most reduced MPO activity (all P<0.01; Fig. 5C). According to the flow cytometry results, the MFI value and percentage of phagocytic positive cells were markedly higher in the ALI + IAXO-102 group and the ATRA + PBS group compared with the ALI + PBS group and the ATRA + IAXO-102 group showed the highest MFI value and percentage of phagocytic positive cells (all $\mathrm{P}<0.01$; Fig. 5D and E). ELISA results revealed that the ALI + IAXO-102 group and ATRA + PBS group presented markedly reduced levels of inflammatory cytokines in plasma and the ATRA + IAXO-102 group exhibited the lowest levels of inflammatory cytokines (all $\mathrm{P}<0.01$; Fig. 5F). Meanwhile, no notable difference in the indicators was found between the ALI + IAXO-102 group and the ATRA + PBS group ( $>0.05)$. In summary, ATRA enhanced macrophage phagocytosis and reduced inflammatory cytokine levels by inhibiting CD14/TLR4 in LPS-induced ALI rats.

\section{Discussion}

ALI is a serious heterogeneous lung disease often accompanied by lung inflammation and alveolar macrophages have been demonstrated to participate in lung inflammation owing to their phagocytosis $(4,29)$. ATRA has been demonstrated to serve a potential regulatory role in multiple cellular processes such as cell activity and inflammation $(30,31)$. The present study demonstrated that ATRA promoted macrophage phagocytosis and reduced inflammation in LPS-induced ALI rats by inhibiting the CD14/TLR4-NF- $\mathrm{BB}$ pathway.

ATRA is a carboxyl form of vitamin A (32) and exerts protective effects against liver and kidney injury $(33,34)$. In addition, ATRA is closely associated with lung diseases $(35,36)$. However, little is known about the specific role of ATRA 

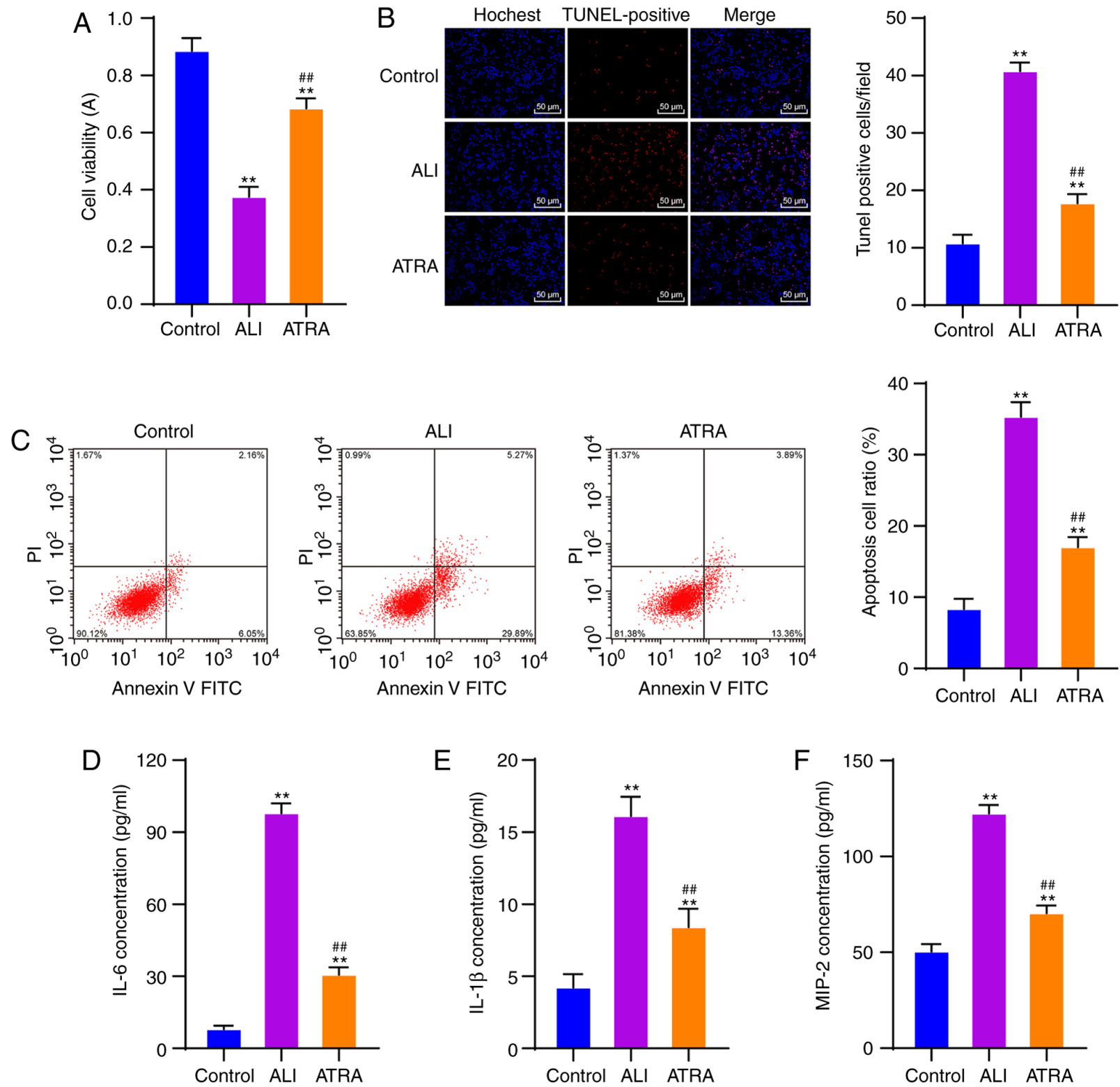

Figure 3. ATRA increases macrophage viability and inhibited apoptosis and decreases inflammatory cytokine levels in ALI rats. (A) CCK-8 assay was used to detect the viability of macrophages in ALI rats. (B) TUNEL and (C) flow cytometry were used to detect the apoptosis of macrophages in ALI rats. (D-F) ELISA kits were used to detect the levels of inflammatory cytokines (D) IL-6, (E) IL-1 $\beta$ and (F) MIP-2 in plasma of rats in each group. The cell experiments were repeated three times independently. Data are expressed as mean \pm standard deviation and analyzed using one-way analysis of variance, followed by Tukey's multiple comparisons test. ${ }^{* *} \mathrm{P}<0.01$ vs. control group; ${ }^{\# \#} \mathrm{P}<0.01$ vs. ALI group. ATRA, all-trans retinoic acid; ALI, acute lung injury; MIP-2, macrophage inflammatory protein- 2 .

in ALI. It has been demonstrated that biomarkers in the plasma and BALF can be used to evaluate ALI severity (37). Decreased lung $\mathrm{W} / \mathrm{D}$ ratio and protein content in BALF, together with increased $\mathrm{PaO}_{2}$, are indicators of attenuated ALI $(38,39)$. According to the findings of the present study, ATRA markedly increased $\mathrm{PaO}_{2}$ in arterial blood and reduced the protein content in BALF and lung W/D ratio in ALI rats, along with effectively alleviating pathological lung injury. In accordance with this, a previous study demonstrated that RA possesses beneficial effects on injured lung tissues following ALI (40). ATRA may help to alleviate transfusion-related ALI (41). According to previous studies $(32,42-44)$, olive oil is usually selected as the control of ATRA treatment. Accordingly, the present study used olive oil as the control of
ATRA treatment. Meanwhile, preliminary experiments were conducted to exclude the possible influence of olive oil treatment on this experiment (Fig. S2). The results of the present study demonstrated that ATRA had protective effects on LPS-induced ALI rats.

It has been demonstrated that macrophages are closely implicated in ALI pathogenesis as a crucial organizer (45). Alveolar macrophages alleviate ALI, which may be attributed to their phagocytic functions (29). In the present study, BALF was collected and alveolar macrophages were isolated and purified. It was found that ALI rats showed notably decreased macrophage phagocytosis, which was then clearly enhanced following ATRA treatment. ATRA efficiently improves the phagocytic function of macrophages (46). The effects of 
A

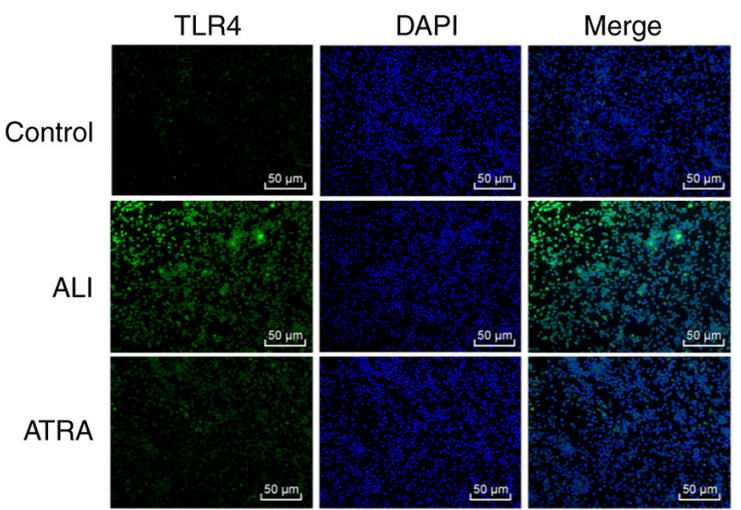

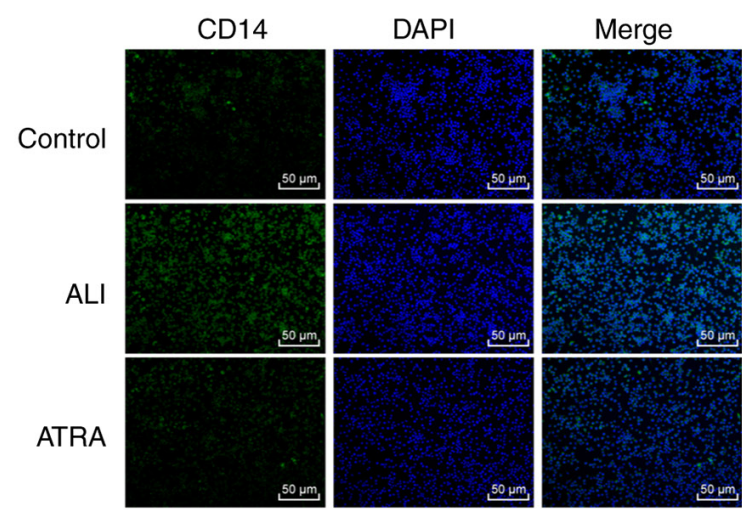

B

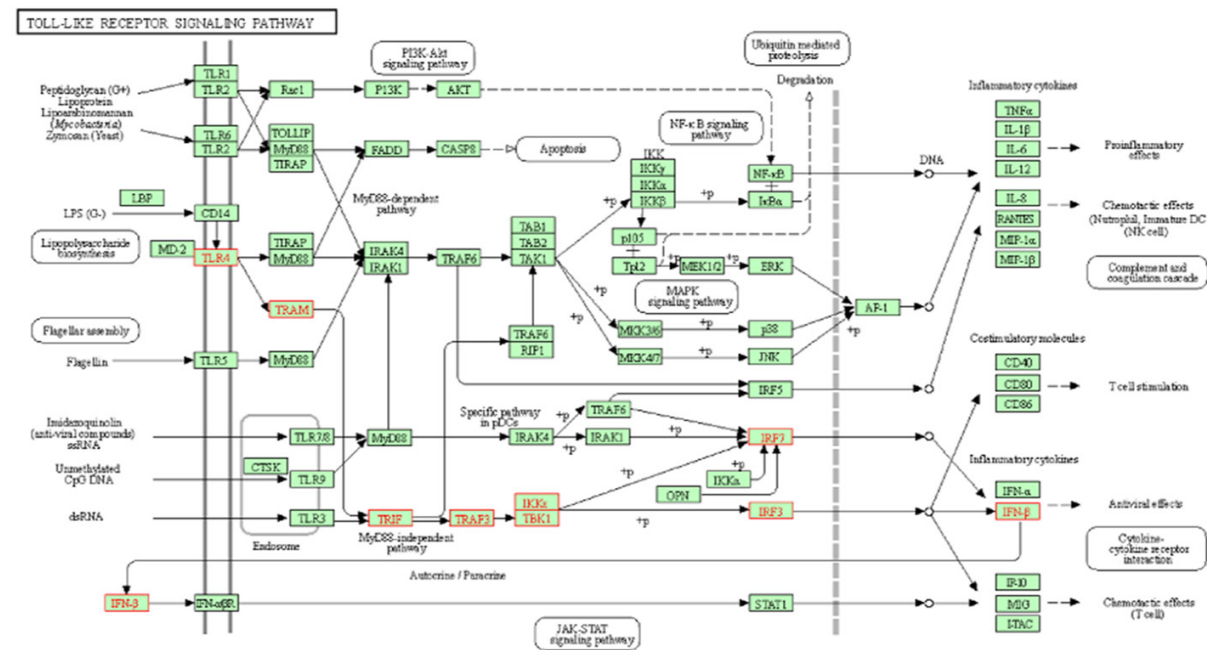

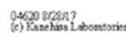

C

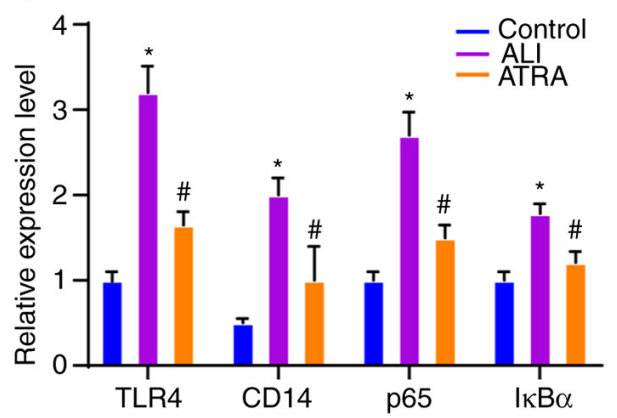

D Control ALI ATRA

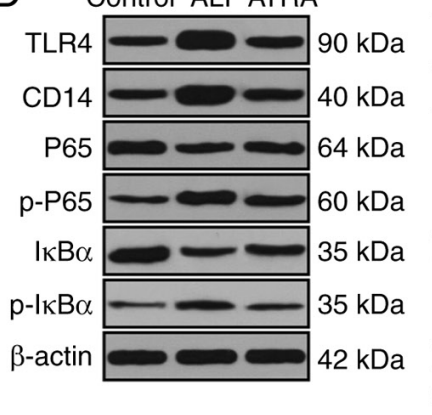

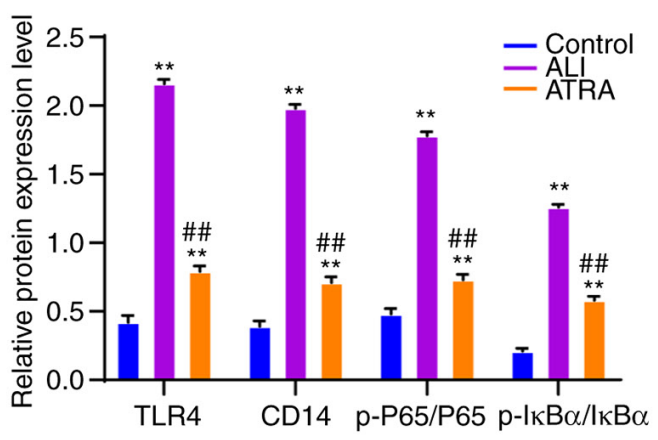

Figure 4. ATRA inhibits CD14/TLR4 upregulation and the downstream NF- $\kappa$ B pathway activation in macrophages in LPS-induced ALI rats. (A) CD14 and TLR4 expressions on macrophage membrane are detected using immunofluorescence. (B) Key pathways in LPS signal transduction through the Kyoto Encyclopedia of Genes and Genomes database (https://www.kegg.jp/). (C) mRNA expressions of TLR4, CD14, P65 and IкB $\alpha$ are detected using reverse

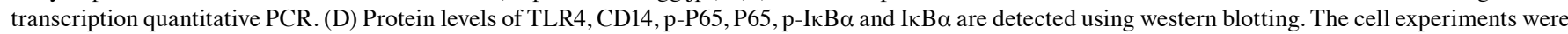
repeated three times independently. Data are expressed as mean \pm standard deviation and analyzed using one-way or two-way analysis of variance, followed by Tukey's multiple comparisons test. ${ }^{*} \mathrm{P}<0.05,{ }^{* *} \mathrm{P}<0.01$ vs. control group; ${ }^{\#} \mathrm{P}<0.05,{ }^{\# \#} \mathrm{P}<0.01$ vs. ALI group. ATRA, all-trans retinoic acid; CD, cluster of differentiation; TLR, Toll-like receptor; LPS, lipopolysaccharide; ALI, acute lung injury; p-, phosphorylated.

ATRA on macrophage viability and apoptosis in ALI rats were further assessed. It was shown that ATRA treatment markedly increased macrophage viability and reduced apoptosis. In agreement with these findings, alveolar macrophages exhibit reduced cell viability and augmented apoptosis rate in LPS-induced ALI (47). Furthermore, macrophage activity is closely associated with the inflammatory response in ALI (48). Hence, levels of inflammatory cytokines (IL-6, IL-1 $\beta$ and MIP-2) were detected in the plasma of ALI rats and were observed to be noticeably reduced in ATRA-treated
ALI rats. A previous study identified that RA helps to reduce levels of proinflammatory cytokines, thereby attenuating lung dysfunction (49). Taken together, the present study confirmed that ATRA increased phagocytosis and promoted the viability and inhibited apoptosis of macrophages as well as decreased inflammation in ALI rats.

Subsequently, the underlying mechanism of ATRA in ALI was explored. ALI may develop as a consequence of LPS binding to its receptors, such as CD14 and TLR4 (50). TLR4 and CD14 serve crucial roles in responding to inflammatory stimuli 

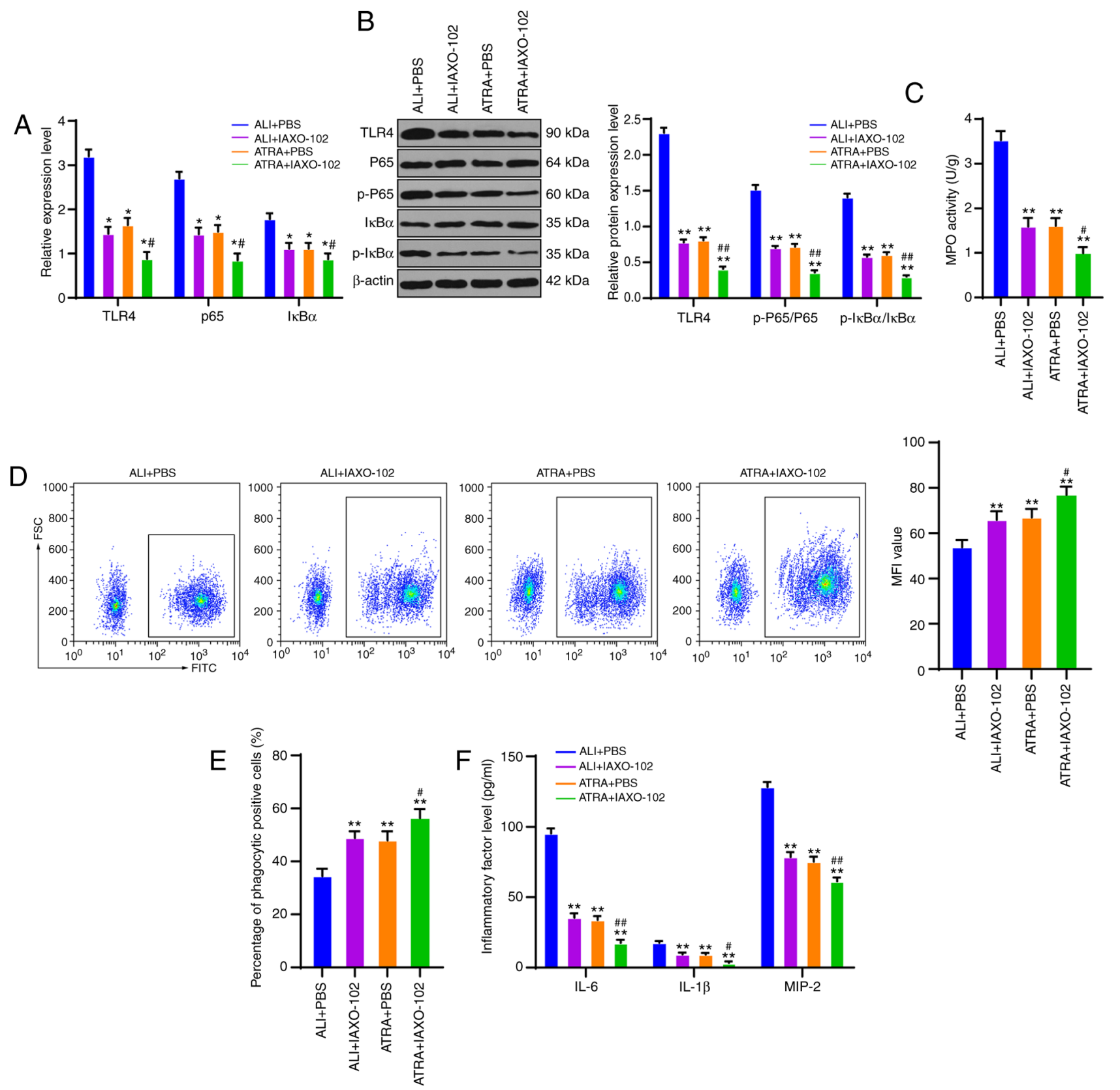

Figure 5. ATRA enhances macrophage phagocytosis and reduces inflammatory cytokine levels via inhibiting CD14/TLR4 expressions in LPS-induced ALI rats. (A) mRNA expressions of TLR4, CD14, P65 and IкB $\alpha$ are detected using reverse transcription quantitative PCR. (B) Western blotting was used to

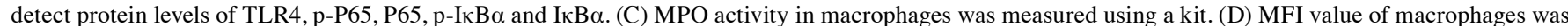
measured using flow cytometry. (E) Percentage of macrophage phagocytic positive cells was determined using flow cytometry. (F) Levels of inflammatory cytokines (IL-6, IL-1 $\beta$ and MIP-2) in rat plasma were detected using ELISA kits. The cell experiments were repeated three times independently. Data were expressed as mean \pm standard deviation and analyzed using one-way or two-way analysis of variance, followed by Tukey's multiple comparisons test. "P $<0.05$, ${ }^{* *} \mathrm{P}<0.01$ vs. the ALI + PBS group; ${ }^{\prime} \mathrm{P}<0.05{ }^{\# \#} \mathrm{P}<0.01$ vs. the ATRA + IAXO-102 group. ATRA, all-trans retinoic acid; CD, cluster of differentiation; TLR, Toll-like receptor; LPS, lipopolysaccharide; ALI, acute lung injury; p-, phosphorylated; MPO, myeloperoxidase; MFI, mean fluorescence intensity; MIP-2, macrophage inflammatory protein-2.

in macrophages $(51,52)$. Based on the results of the present study, CD14 and TLR4 expression were clearly upregulated in ALI rat macrophages and notably decreased following ATRA treatment. A previous study showed that CD14 and TLR4 expression is increased in LPS-induced ALI (53). RA receptor agonists help to inhibit CD14 and TLR4 signaling and then suppress the NF- $\kappa \mathrm{B}$ pathway (44). It has been demonstrated that TLR4 mediates the NF- $\kappa \mathrm{B}$ pathway in macrophages and they serve a crucial role in ALI $(27,28,52)$. The present study confirmed that the $\mathrm{NF}-\kappa \mathrm{B}$ pathway was notably activated in ALI and ATRA treatment effectively inhibited NF- $\kappa$ B pathway activation. A previous study demonstrated that ATRA administration suppresses the TLR4/NF- $\kappa \mathrm{B}$ inflammatory pathway in diabetic nephropathy (54). In addition, according to the functional rescue experiment results of the present study, inhibition of TLR4 markedly enhanced the efficacy of ATRA on ALI, as manifested by notably increased macrophage phagocytosis and decreased inflammation in ALI rats. Similarly, TLR4 downregulation in the lung is closely associated with suppressed inflammation and lung injury (55). 
Suppression of TLR4 helps RA to attenuate the LPS-induced inflammatory response (56). Taken together, ATRA inhibited CD14/TLR4 expression and the downstream NF- $\mathrm{BB}$ pathway activation in macrophages, thereby enhancing macrophage phagocytosis and decreasing inflammation in ALI.

Overall, the present study found that ATRA promoted macrophage phagocytosis and decreased inflammation via inhibition of CD14/TLR4 expression and NF- $\kappa B$ pathway activation in LPS-induced ALI. ATRA could affect macrophage function, and it exerted an effect on the expression of CD14/TLR4 and alleviated the process of ALI. These results revealed a novel ATRA-based therapy for ALI patients. Although the present study provides therapeutic value for ALI treatment, the experimental results and clinical application need further validation.

\section{Acknowledgments}

Not applicable.

\section{Funding}

No funding was received.

\section{Availability of data and materials}

The datasets used and/or analyzed during the current study are available from the corresponding author on reasonable request.

\section{Authors' contributions}

SL made substantial contributions to the conception of the present study. YL and JL performed the experiments and wrote the manuscript. HL contributed to the design of the present study and interpreted the data. SL and HL confirm the authenticity of all the raw data. All authors read and approved the final manuscript.

\section{Ethics approval and consent to participate}

All animal procedures were performed in accordance with the guidelines of the Animal Ethics Committee of Shanxi Provincial People's Hospital and ethics approval was received (approval no. 2019-56). Significant efforts were made to minimize animal numbers and their suffering.

\section{Patient consent for publication}

Not applicable.

\section{Competing interests}

The authors declare that they have no competing interests.

\section{References}

1. Elicker BM, Jones KT, Naeger DM and Frank JA: Imaging of acute lung injury. Radiol Clin North Am 54: 1119-1132, 2016.

2. Fröhlich S, Boylan J and McLoughlin P: Hypoxia-induced inflammation in the lung: A potential therapeutic target in acute lung injury? Am J Respir Cell Mol Biol 48: 271-279, 2013.
3. Gouda MM and Bhandary YP: Acute lung injury: IL-17A-mediated inflammatory pathway and its regulation by curcumin. Inflammation 42: 1160-1169, 2019.

4. Mokra D, Mikolka P, Kosutova P and Mokry J: Corticosteroids in acute lung injury: The dilemma continues. Int J Mol Sci 20: $4765,2019$.

5. Perl M, Lomas-Neira J, Venet F, Chung CS and Ayala A: Pathogenesis of indirect (secondary) acute lung injury. Expert Rev Respir Med 5: 115-126, 2011.

6. Barbalho SM, de Goulart RA and Batista G: Vitamin A and inflammatory bowel diseases: From cellular studies and animal models to human disease. Expert Rev Gastroenterol Hepatol 13: 25-35, 2019.

7. Chen F, Cao Y, Qian J, Shao F, Niederreither K and Cardoso WV: A retinoic acid-dependent network in the foregut controls formation of the mouse lung primordium. J Clin Invest 120: 2040-2048, 2010.

8. Nagpal I and Wei LN: All-trans retinoic acid as a versatile cytosolic signal modulator mediated by CRABP1. Int J Mol Sci 20: $3610,2019$.

9. Sierra-Mondragon E, Rodriguez-Munoz R, Namorado-Tonix C, Molina-Jijon E, Romero-Trejo D, Pedraza-Chaverri J and Reyes JL: All-trans retinoic acid attenuates fibrotic processes by downregulating TGF- $\beta 1 / \mathrm{Smad} 3$ in early diabetic nephropathy. Biomolecules 92: 525, 2019.

10. Yu D, Cai SY, Mennone A, Vig P and Boyer JL: Cenicriviroc, a cytokine receptor antagonist, potentiates all-trans retinoic acid in reducing liver injury in cholestatic rodents. Liver Int 38: $1128-1138,2018$

11. Uniyal S, Dhasmana A, Tyagi A and Muyal JP: ATRA reduces inflammation and improves alveolar epithelium regeneration in emphysematous rat lung. Biomed Pharmacother 108: 1435-1450, 2018.

12. Kawasaki T, Ito K, Miyata H, Akira S and Kawai T: Deletion of PIKfyve alters alveolar macrophage populations and exacerbates allergic inflammation in mice. EMBO J 36: 1707-1718, 2017.

13. Huang P, Wei S, Huang W, Wu P, Chen S, Tao A, Wang H, Liang Z, Chen R, Yan J and Zhang Q: Hydrogen gas inhalation enhances alveolar macrophage phagocytosis in an ovalbumin-induced asthma model. Int Immunopharmacol 74: 105646, 2019.

14. Moser EK, Field NS and Oliver PM: Aberrant Th2 inflammation drives dysfunction of alveolar macrophages and susceptibility to bacterial pneumonia. Cell Mol Immunol 15: 480-492, 2018.

15. Bonhomme D, Santecchia I, Vernel-Pauillac F, Caroff M, Germon P, Murray G, Adler B, Boneca IV and Werts C: Leptospiral LPS escapes mouse TLR4 internalization and TRIFassociated antimicrobial responses through $\mathrm{O}$ antigen and associated lipoproteins. PLoS Pathog 16: e1008639, 2020.

16. Wu X, Kong Q, Zhan L, Qiu Z, Huang Q and Song X: TIPE2 ameliorates lipopolysaccharide-induced apoptosis and inflammation in acute lung injury. Inflamm Res 68: 981-992, 2019.

17. Intengan HD and Smyth DD: Renal alpha 2a/d-adrenoceptor subtype function: Wistar as compared to spontaneously hypertensive rats. Br J Pharmacol 121: 861-866, 1997.

18. Sun A, Wang W, Ye X, Wang Y, Yang X, Ye Z, Sun X and Zhang C: Protective effects of methane-rich saline on rats with lipopolysaccharide-induced acute lung injury. Oxid Med Cell Longev 2017: 7430193, 2017.

19. Xia H, Ge Y, Wang F, Ming Y, Wu Z, Wang J, Sun S, Huang S, Chen M, Xiao W and Yao S: Protectin DX ameliorates inflammation in sepsis-induced acute lung injury through mediating PPAR $\gamma / \mathrm{NF}-\kappa \mathrm{B}$ pathway. Immunol Res 68: 280-288, 2020.

20. Li Q, Ran Q, Sun L, Yin J, Luo T, Liu L, Zhao Z, Yang Q, Li Y, Chen Y, et al: Lian Hua Qing Wen Capsules, a potent epithelial protector in acute lung injury model, block proapoptotic communication between macrophages, and alveolar epithelial cells. Front Pharmacol 11: 522729, 2020

21. Yi R, Wei Y, Tan F, Mu J, Long X, Pan Y, Liu W and Zhao X: Antioxidant capacity-related preventive effects of shoumei (Slightly Fermented Camellia sinensis) polyphenols against hepatic injury. Oxid Med Cell Longev 2020: 9329356, 2020.

22. Livak KJ and Schmittgen TD: Analysis of relative gene expression data using real-time quantitative PCR and the 2(-Delta Delta C(T)) method. Methods 25: 402-408, 2001.

23. Li B, Gao MH, Lv CY, Yang P and Yin QF: Study of the synergistic effects of all-transretinoic acid and C-phycocyanin on the growth and apoptosis of A549 cells. Eur J Cancer Prev 25: 97-101, 2016. 
24. Miao J, Ye S, Lan J, Ye P, Wen Q, Mei L, Liu X, Lin J, Zhou X, Du S, et al: Nuclear HMGB1 promotes the phagocytic ability of macrophages. Exp Cell Res 393: 112037, 2020.

25. Wang C, Petriello MC, Zhu B and Hennig B: PCB 126 induces monocyte/macrophage polarization and inflammation through AhR and NF-кB pathways. Toxicol Appl Pharmacol 367: 71-81, 2019.

26. Leláková V, Beraud-Dufour S, Hošek J, Šmejkal K, Prachyawarakorn V, Pailee P, Widmann C, Václavík J, Coppola T, Mazella J, et al: Therapeutic potential of prenylated stilbenoid macasiamenene $\mathrm{F}$ through its anti-inflammatory and cytoprotective effects on LPS-challenged monocytes and microglia. J Ethnopharmacol 263: 113147, 2020.

27. Liu TY, Zhao LL, Chen SB, Hou BC, Huang J, Hong X, Qing L, Fang Y and Tao Z: Polygonatum sibiricum polysaccharides prevent LPS-induced acute lung injury by inhibiting inflammation via the TLR4/Myd88/NF- $\mathrm{B}$ pathway. Exp Ther Med 20: 3733-3739, 2020.

28. Zhang Y,Zhu Y, Gao G and Zhou Z: Knockdown XIST alleviates LPS-induced WI-38 cell apoptosis and inflammation injury via targeting miR-370-3p/TLR4 in acute pneumonia. Cell Biochem Funct 37: 348-358, 2019.

29. Mohning MP, Thomas SM, Barthel L, Mould KJ, McCubbrey AL Frasch SC, Bratton DL, Henson PM and Janssen WJ: Phagocytosis of microparticles by alveolar macrophages during acute lung injury requires MerTK. Am J Physiol Lung Cell Mol Physiol 314: L69-L82, 2018.

30. Liu ZM, Wang KP, Ma J and Zheng SG: The role of all-trans retinoic acid in the biology of Foxp3+ regulatory $\mathrm{T}$ cells. Cell Mol Immunol 12: 553-557, 2015.

31. Zhou TB, Ou C, Jiang ZP, Xiong MR and Zhang F: Potential signal pathway between all-trans retinoic acid and LMX1B in hypoxia-induced renal tubular epithelial cell injury. J Recept Signal Transduct Res 36: 53-56, 2016.

32. Amengual J, Ribot J, Bonet ML and Palou A: Retinoic acid treatment increases lipid oxidation capacity in skeletal muscle of mice. Obesity (Silver Spring) 16: 585-591, 2008.

33. Elshal M, Abu-Elsaad N, El-Karef A and Ibrahim T: Retinoic acid modulates IL-4, IL-10 and MCP-1 pathways in immune mediated hepatitis and interrupts CD4+ T cells infiltration. In Immunopharmacol 75: 105808, 2019.

34. Wu J, Wan X, Zhang H, Li W, Ma M, Pan B, Liang X and Cao C: Retinoic acid attenuates contrast-induced acute kidney injury in a miniature pig model. Biochem Biophys Res Commun 512: 163-169, 2019.

35. Leem AY, Shin MH, Douglas IS, Song JH, Chung KS, Kim EY, Jung JY, Kang YA, Chang J, Kim YS and Park MS: All-trans retinoic acid attenuates bleomycin-induced pulmonary fibrosis via downregulating EphA2-EphrinA1 signaling. Biochem Biophys Res Commun 491: 721-726, 2017.

36. Zhang Y, Zhao J, Sun J, Huang L and Li Q: Targeting lung cancer initiating cells by all-trans retinoic acid-loaded lipid-PLGA nanoparticles with CD133 aptamers. Exp Ther Med 16: 4639-4649, 2018.

37. Mokra D and Kosutova P: Biomarkers in acute lung injury. Respir Physiol Neurobiol 209: 52-58, 2015.

38. Liu W, Liu K, Zhang S, Shan L and Tang J: Tetramethylpyrazine showed therapeutic effects on sepsis-induced acute lung injury in rats by inhibiting endoplasmic reticulum stress protein kinase RNA-Like endoplasmic reticulum kinase (PERK) signaling-induced apoptosis of pulmonary microvascular endothelial cells. Med Sci Monit 24: 1225-1231, 2018.

39. Zhou H, Li F, Niu JY, Zhong WY, Tang MY, Lin D, Cui HH, Huang XH, Chen YY, Wang HY and Tu YS: Ferroptosis was involved in the oleic acid-induced acute lung injury in mice. Sheng Li Xue Bao 71: 689-697, 2019.

40. Yang C, Yang X, Du J, Wang H, Li H, Zeng L, Gu W and Jiang J: Retinoic acid promotes the endogenous repair of lung stem/progenitor cells in combined with simvastatin after acute lung injury: A stereological analysis. Respir Res 16: 140, 2015.
41. Jeddi R, Mansouri R, Kacem K, Gouider E, Abid HB, Belhadjali Z and Meddeb B: Transfusion-related acute lung injury (TRALI) during remission induction course of acute myeloid leukemia: A possible role for all-transretinoic-acid (ATRA)? Pathol Biol (Paris) 57: 500-502, 2009.

42. Uniyal S, Tyagi AK and Muyal JP: All trans retinoic acid (ATRA) progresses alveolar epithelium regeneration by involving diverse signalling pathways in emphysematous rat. Biomed Pharmacother 131: 110725, 2020.

43. Seifart C, Muyal JP, Plagens A, Yildirim AÖ, Kohse K, Grau V, Sandu S, Reinke C, Tschernig T, Vogelmeier C and Fehrenbach H: All-trans retinoic acid results in irregular repair of septa and fails to inhibit proinflammatory macrophages. Eur Respir J 38: 425-439, 2011.

44. Ramirez-Moreno A, Escorza MA, Garza RG, Hady K, Valenzuela AM, Marszalek JE, Sharara-Núñez I and Delgadillo-Guzmán D: All-trans retinoic acid improves pancreatic cell proliferation on induced type 1 diabetic rats. Fundam Clin Pharmacol 34: 345-351, 2020.

45. Chen X, Tang J, Shuai W, Meng J, Feng J and Han Z: Macrophage polarization and its role in the pathogenesis of acute lung injury/acute respiratory distress syndrome. Inflamm Res 69: $883-895,2020$

46. Lo HM, Wang SW, Chen CL, Wu PH and Wu WB: Effects of all-trans retinoic acid, retinol, and beta-carotene on murine macrophage activity. Food Funct 5: 140-148, 2014.

47. Zhang Z, Zhang Y and Zhou R: Loss of annexin A5 expression attenuates the lipopolysaccharide-induced inflammatory response of rat alveolar macrophages. Cell Biol Int 44: 391-401, 2020.

48. Huang X, Xiu H, Zhang S and Zhang G: The role of macrophages in the pathogenesis of ALI/ARDS. Mediators Inflamm 2018: $1264913,2018$.

49. James ML, Ross AC, Nicola T, Steele $C$ and Ambalavanan N: VARA attenuates hyperoxia-induced impaired alveolar development and lung function in newborn mice. Am J Physiol Lung Cell Mol Physiol 304: L803-L812, 2013.

50. Jeyaseelan S, Chu HW, Young SK, Freeman MW and Worthen GS: Distinct roles of pattern recognition receptors CD14 and toll-like receptor 4 in acute lung injury. Infect Immun 73: 1754-1763, 2005.

51. Chen Z, Ding X, Jin S, Pitt B, Zhang L, Billiar T and Li Q: WISP1- $\alpha v \beta 3$ integrin signaling positively regulates TLR-triggered inflammation response in sepsis induced lung injury. Sci Rep 6: 28841, 2016.

52. Ren W, Wang Z, Hua F and Zhu L: Plasminogen activator inhibitor-1 regulates LPS-induced TLR4/MD-2 pathway activation and inflammation in alveolar macrophages. Inflammation 38: 384-393, 2015.

53. Ma L, Wu XY, Zhang LH, Chen WM, Uchiyama A, Mashimo T and Fujino Y: Propofol exerts anti-inflammatory effects in rats with lipopolysaccharide-induced acute lung injury by inhibition of CD14 and TLR4 expression. Braz J Med Biol Res 46: 299-305, 2013.

54. Sierra-Mondragon E, Molina-Jijon E, Namorado-Tonix C, Rodriguez-Munoz R, Pedraza-Chaverri J and Reyes JL: All-trans retinoic acid ameliorates inflammatory response mediated by TLR4/NF- $\kappa$ B during initiation of diabetic nephropathy. J Nutr Biochem 60: 47-60, 2018

55. Tang SE, Wu SY, Chu SJ, Tzeng YS, Peng CK, Lan CC, Perng WC, Wu CP and Huang KL: Pre-treatment with ten-minute carbon dioxide inhalation prevents lipopolysaccharide-induced lung injury in mice via down-regulation of toll-like receptor 4 expression. Int J Mol Sci 20: 6293, 2019.

56. Gu B, Miao J, Fa Y, Lu J and Zou S: Retinoic acid attenuates lipopolysaccharide-induced inflammatory responses by suppressing TLR4/NF-kappaB expression in rat mammary tissue. Int Immunopharmacol 10: 799-805, 2010.

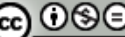

This work is licensed under a Creative Commons Attribution-NonCommercial-NoDerivatives 4.0 International (CC BY-NC-ND 4.0) License. 